\title{
Simultaneous Spatial and Spectral Transparency in Ultralong Fiber Lasers
}

\author{
J. D. Ania-Castañón, ${ }^{1,2}$ V. Karalekas, ${ }^{1}$ P. Harper, ${ }^{1}$ and S. K. Turitsyn ${ }^{1}$ \\ ${ }^{1}$ Photonics Research Group, School of Engineering and Applied Science, Aston University, Birmingham B4 7ET, United Kingdom \\ ${ }^{2}$ Instituto de Óptica, CSIC, Serrano 121, 28006 Madrid, Spain
}

(Received 30 May 2008; revised manuscript received 5 August 2008; published 15 September 2008)

\begin{abstract}
We demonstrate that ultralong Raman lasers can be used to generate a transmission medium with simultaneous transparency over the spatial and the spectral domains. Numerical calculations show this cross-domain transparency to be preserved when the medium is used for transmitting high-intensity signals, which makes ultralong lasers an ideal experimental test bed for the study of multifrequency nonlinear interactions in optical fiber waveguides. Full spatiospectral transparency is experimentally obtained over a $20 \mathrm{~nm} \times 20 \mathrm{~km}$ window.
\end{abstract}

DOI: 10.1103/PhysRevLett.101.123903

PACS numbers: 42.81.Dp, 42.55.Ye, 42.65.Dr

Introduction.-An ideal transmission medium that could allow the nonmodified transfer of information from a transmitter to a receiver is the ultimate and likely unreachable dream of telecommunications. In practice, any real transmission medium introduces distortions into the signal that can be either recoverable (e.g., dispersive broadening) or not fully removable (e.g., noise). Optical fiber waveguides are an example of a medium with numerous attractive features such as low loss, large bandwidth, and low cost. Despite the reduced loss, optical fiber transmission takes place over extremely long distances, requiring periodic signal amplification, which introduces noise. An adequate combination of classical optical fiber technology with novel techniques might further improve the properties of the transmission medium. In this Letter we discuss and demonstrate the possibility of creating transmission media with cross-domain (spatial and spectral) transparency (effective zero local attenuation across the plane) and reduced noise by applying ultralong Raman fiber laser technology.

Fiber cavity lasers [1-3], in which the active gain medium is a doped or pure silica optical fiber waveguide with fiber Bragg grating reflectors commonly used to provide a resonant cavity [4], have attracted much attention as they exhibit multiple applications and have seen a rapid technological development over the past few years. Fiber lasers are successfully employed in applied areas such as telecommunications, material processing, spectroscopy, or medicine, as well as having become a very important tool in basic research, where they are used, for example, in laser cooling, slow and fast light generation, and the study of fundamental nonlinear science. Recently, our group proposed [5] and demonstrated [6] the concept of ultralong Raman lasers (URFLs), which take advantage of the reduced attenuation and excellent confinement of standard telecommunication optical fiber waveguides to create laser cavities with lengths ranging from a few $\mathrm{km}$ to more than a $100 \mathrm{~km}$ [7], using the Raman effect [4,8] to provide the gain in the fiber. The extreme length of these resonant cavities makes the physical behavior of URFLs genuinely different from that of traditional fiber lasers, and opens the door to a multiplicity of potentially interesting applications, from the design of long-distance repeaterless communication systems [9] to the generation of dense frequency combs [10] or the creation of real-life nonlinear integrable optical devices in which propagation can be described through the traditional, integrable nonlinear Schrödinger equation thanks to the cancellation of the non-Hamiltonian dissipative term. One of the most important features of URFLs lies in their ability to perform as virtually lossless, low-noise optical fiber links to be used in long-distance communications or optical processing. Furthermore, it has been shown [9] that long URFL-based links are able to provide gain over a broad spectral bandwidth.

In this Letter, we demonstrate for the first time that virtual cross-domain spatiospectral transparency, with close-to-zero effective attenuation across the frequencydistance plane, is achievable with standard fiber URFL links of moderate lengths (of up to $25 \mathrm{~km}$ ). Such fiber spans act, in terms of intensity variations, as perfectly transparent media for a broad range of transmitted intensities and could provide, for example, the basis for a new class of multiwavelength optical processing devices in which interchannel nonlinear interactions can be exploited within a nondissipative, integrable framework. Moreover, such a transparent medium can be advantageous both for linear and nonlinear transmission and processing regimes, thanks to the reduction in amplified spontaneous emission noise caused by the mutual cancellation of the dissipative and gain terms along the spatiospectral plane. In this Letter, we present the key features of the achieved crossdomain transparency and study theoretically and experimentally the dependence of the characteristics of such a medium on both transmitted power and cavity length.

Theory and experimental setup.-It has previously been shown $[6,7]$ that the behavior of an URFL can be very accurately described by numerically solving the steadystate laser equations, as long as all important effects such 
as pump depletion, amplified spontaneous emission, and double-Rayleigh scattering noise are properly taken into account. As we will see below, our simulations once again show excellent agreement with the experiment and allow us to extrapolate the behavior of our scheme at high powers not reachable by our experimental setup. Although a numerical approach is necessary to accurately solve the problem, an analytical formulation is interesting for the sake of clarity. Following the general steady-state equations for a bidirectionally pumped URFL link [6], neglecting noise terms, assuming negligible spectral broadening of the first Stokes, and taking into account the contribution of the primary pump to signal gain (usually neglected when cascading between Stokes waves, but not here as our signal is multiwavelength), it is easy to arrive at an expression for the spatial derivative of the signal power:

$$
\begin{aligned}
\frac{d P_{S}(\nu, z)}{d z}=\{ & -\alpha(\nu)+g_{R}\left(\mu_{2}, \nu\right)\left[P_{2}^{+}\left(\mu_{2}, z\right)+P_{2}^{-}\left(\mu_{2}, z\right)\right] \\
& \left.+g_{R}\left(\mu_{1}, \nu\right)\left[P_{1}^{+}\left(\mu_{1}, z\right)+P_{1}^{-}\left(\mu_{1}, z\right)\right]\right\} P_{S}(\nu, z),
\end{aligned}
$$

where + and - represent, respectively, copropagating and counterpropagating directions, $\nu$ represents the frequency of the particular signal component, $g_{R}(\mu, \nu)$ represents the fiber Raman gain coefficient from frequency $\mu$ to frequency $\nu$, and $P_{1}\left(\mu_{1}, z\right)$ and $P_{2}\left(\mu_{2}, z\right)$ represent, respectively, the primary pump power and the power of the trapped first Stokes component, each assumed monochromatic. From (1) it follows that for cross-domain transparency to occur for our signal in the transmission fiber, a continuous interval $\left[f_{1}, f_{2}\right]$ in the frequency domain must exist such that for all $(\nu, z) \in\left[f_{1}, f_{2}\right] x[0, L]$ :

$$
\begin{aligned}
\alpha(\nu) \approx & g_{R}\left(\mu_{2}, \nu\right)\left[P_{2}^{+}\left(\mu_{2}, z\right)+P_{2}^{-}\left(\mu_{2}, z\right)\right] \\
& +g_{R}\left(\mu_{1}, \nu\right)\left[P_{1}^{+}\left(\mu_{1}, z\right)+P_{1}^{-}\left(\mu_{1}, z\right)\right],
\end{aligned}
$$

where $L$ is the length of the URFL transmission link.

In general, $P_{1}$ and $P_{2}$ are both dependent on each other and on $P_{S}$, so expression (1) cannot be rearranged in a more explicit analytical form in terms of the initial values of $P_{S}$, but it is possible to do so by assuming that neither $P_{1}$ nor $P_{2}$ suffer pump depletion from the signal (very low signal approximation), and thus are not dependent on $P_{S}$. Under this consideration, it is straightforward to rewrite (1) as

$$
P_{S}(\nu, Z)=P_{S}(\nu, 0) \exp \left(-\alpha_{\text {eff }}(\nu, Z) Z\right)
$$

where

$$
\begin{aligned}
\alpha_{\mathrm{eff}}(\nu, Z)= & \frac{1}{Z} \int_{0}^{Z}\left\{\alpha(\nu)-g_{R}\left(\mu_{2}, \nu\right)\right. \\
& \times\left[P_{2}^{+}\left(\mu_{2}, z\right)+P_{2}^{-}\left(\mu_{2}, z\right)\right] \\
& \left.-g_{R}\left(\mu_{1}, \nu\right)\left[P_{1}^{+}\left(\mu_{1}, z\right)+P_{1}^{-}\left(\mu_{1}, z\right)\right]\right\} d z
\end{aligned}
$$

represents the effective gain-loss coefficient, which must be zero in order to achieve transparency. From (4) it becomes evident that condition (2) is equivalent to achieving $\alpha_{\text {eff }}(\nu, Z)=0$ over the specified spatiospectral domain. Please note that the undepleted approximation can be expected to give accurate results only for $P_{S}<10^{-2} P_{i}$, where $i$ can be either 1 or 2 .

The URFL links utilized in our experiment follow the original design [3] depicted schematically in Fig. 1(a). Two Raman pump lasers with central wavelengths of 1365.4 and $1364.2 \mathrm{~nm}$, respectively, for the right and left pump were used as primary pumps. Two fiber Bragg gratings (FBGs) with reflectivities of $\sim 95 \%$ at $1455 \mathrm{~nm}$ were utilized to trap the radiation of the Stokes wave, generating a cavity in the standard single-mode optical fiber link. FBGs reflectivity was chosen to be high in order to reduce losses in the system and increase power efficiency in our experiment, although reflectivity is in itself an extra optimization parameter that can be finely adjusted to further reduce signal power excursion [10], at the cost of increased pump power. FWHM bandwidth of the FBGs was $\sim 125 \mathrm{GHz}(\sim 1 \mathrm{~nm})$. When the power of the primary pumps is above the threshold necessary for stimulated Raman scattering to overcome the fiber attenuation at the central wavelength of the gratings, the whole span becomes an ultralong laser and a dynamically stable secondary bidirectional pump at $1455 \mathrm{~nm}$ is generated in the cavity. As a result, if a signal is to be transmitted in the $1550 \mathrm{~nm}$ region (the Raman Stokes wavelength for the 1455 pump in silica fiber), it can be efficiently amplified with a nearly constant gain that closely matches fiber attenuation along the length of the cavity. In our measurement setup, shown schematically in Fig. 1(b), a tunable laser source was used to provide a variable wavelength probe signal whose power evolution along the transmission line was then monitored using a standard optical time-domain reflectometer

a)

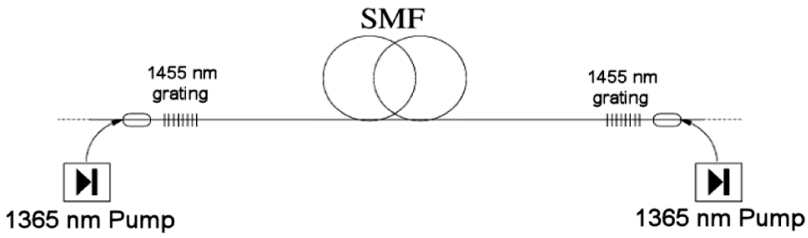

b)

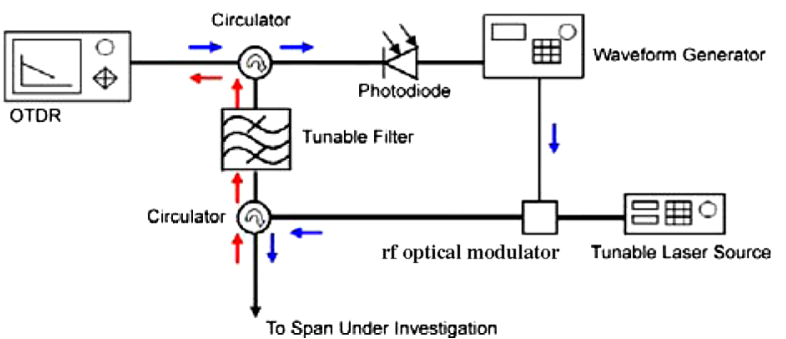

FIG. 1 (color online). (a) Typical URFL cavity. SMF is "standard single mode fiber." (b) Schematic representation of our OTDR setup. 
(OTDR), with pulses of $2 \mu$ s duration and peak power of $\sim 34 \mathrm{~mW}$, with a repetition rate of $1850 \mathrm{~Hz}$. Average signal power was $\sim 0.126 \mathrm{~mW}$. The tunable laser output was swept across a $20 \mathrm{~nm}$ bandwidth between 1540 and $1560 \mathrm{~nm}$. Our signal powers were much lower than the generated Stokes wave power, so that operation takes place with no noticeable depletion of the $1455 \mathrm{~nm}$ component.

Results and discussion.-To provide a meaningful comparison between the transparency performance of URFLs with different cavity lengths, we introduce the dimensionless power excursion parameter $\delta P$, defined as the quotient between the maximal value of signal power excursion $\Delta P$ in the whole spatial-spectral window and the total attenuation in the span, both in $\mathrm{dB} . \delta P=\max [\Delta P(\lambda, z) /(\alpha L)]$, where $\alpha \sim 0.2 \mathrm{~dB} / \mathrm{km}$ over the whole bandwidth of our spectral window. $\delta P$ is a figure of merit indicating how closely the local attenuation of the fiber can be compensated by the Raman gain over the length of the fiber span and the wavelength range measured, relative to the total loss of the span. A value of zero corresponds to no variation in signal power along the fiber, whereas a value of 1 would correspond to lumped amplification at the end of the span, or in general to a complete lack of distributed gain.

Figures 2 and 3 show, respectively, the predicted and the experimental spectral-spatial response of a $20 \mathrm{~km}$ URFL transmission link. For experimental convenience, the spectrum is monitored in terms of wavelength instead of frequencies. From both figures it is obvious that the $20 \mathrm{~km}$ URFL constitutes a fully transparent transmission link, in which the combined action of the two primary pumps and the $1455 \mathrm{~nm}$ laser wave can provide constant amplification to a number of transmitted signals over a bandwidth as broad as $20 \mathrm{~nm}$. For a low-powered signal such as the one used to obtain our OTDR traces, total required $1365 \mathrm{~nm}$ pump power is $370 \mathrm{~mW}$ and the combined + and - power of the $1455 \mathrm{~nm}$ Stokes wave at either end of the cavity is $\sim 130 \mathrm{~mW}$ for full transparency. Please note that the noise in the experimental trace (Fig. 3) is inherent to the OTDR measurements and of the same order as the device precision. The total absolute signal power variation measured along the transmission link is $<0.4 \mathrm{~dB}$, in excellent agree-

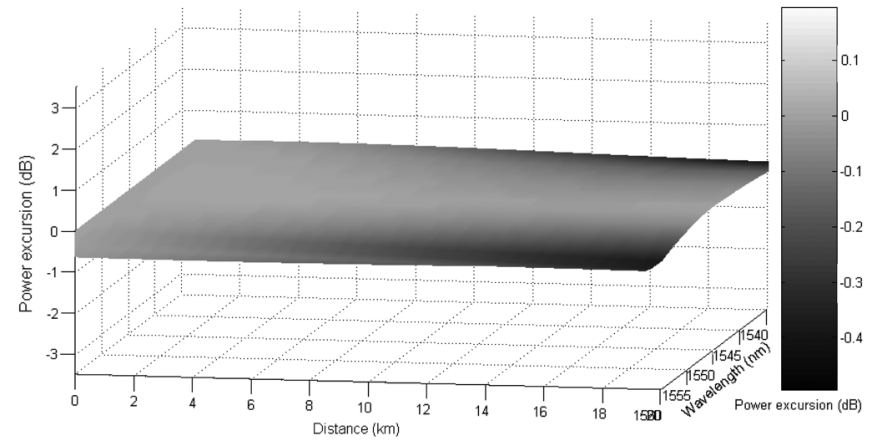

FIG. 2. Numerical signal power excursion in the spectralspatial plane for a $20 \mathrm{~km}$ link. The wavelength range is $20 \mathrm{~nm}$. ment with the theoretical prediction of $0.35 \mathrm{~dB}(\sim 7.8 \%$ intensity) variation over the $1540-1560 \mathrm{~nm}$ range. Variation over the central $10 \mathrm{~nm}$ range between 1545 and $1555 \mathrm{~nm}$ is even smaller, of the order of $0.14 \mathrm{~dB}(\sim 3.1 \%)$ according to the theory, and below the precision of our OTDR setup.

The predicted behavior of these short transparent links of the order of $25 \mathrm{~km}$ or less when used at higher signal powers is very interesting and highly important for their application in nonlinear optical processing. The extremely low power excursion in the spatiospectral plane implies an effective cancellation of the non-Hamiltonian attenuation term, meaning that a multiwavelength transmission system using our transparent links could potentially be described by an integrable set of equations and support, for example, transmission of exact soliton solutions at several wavelengths simultaneously, as long as the link supports high signal powers. This capability is fully confirmed by numerical simulations, which show that the total power excursion actually reduces as input signal power increases, as a side effect of the depletion of the $1455 \mathrm{~nm}$ component. Pump depletion is proportional to the product of pump and signal power, hence being higher close to the extremes of the cavity. This induces an effect on signal transmission akin to that of the lowering of effective reflectivity, which, in our particular case, reduces power excursion as observed in [11]. In addition, the required increase on pump power leads to a higher direct gain from the $1365 \mathrm{~nm}$ pumps to the signal, which further contributes to flattening the spectrum. Indeed, power excursion over $20 \mathrm{~nm}$ is expected to be lowered in our sample $20 \mathrm{~km}$ cavity to just $0.2 \mathrm{~dB}$ $(\sim 4.5 \%)$ over the whole $20 \mathrm{~nm}$ window, as the average signal power is increased to $50 \mathrm{~mW}$ and beyond. These beneficial effects peak at about $160 \mathrm{~mW}$ average signal power (requiring $734 \mathrm{~mW}$ pump power), after which further signal power increases lead to higher power excursions.

Numerical modeling also confirms that this advantage is not present for longer cavities of the kind more likely to be used for long-distance transmission, in which the average power of the Stokes wave is lower and depletion has a

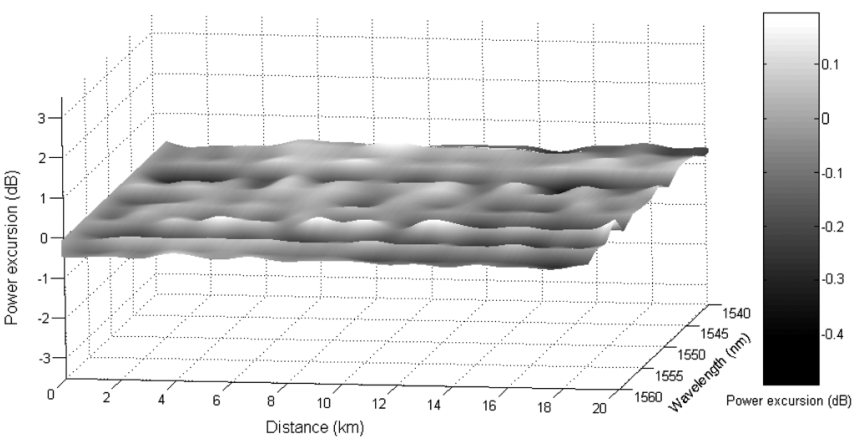

FIG. 3. Experimental signal power excursion in the spectralspatial plane for a $20 \mathrm{~km}$ link. The wavelength range is $20 \mathrm{~nm}$. 

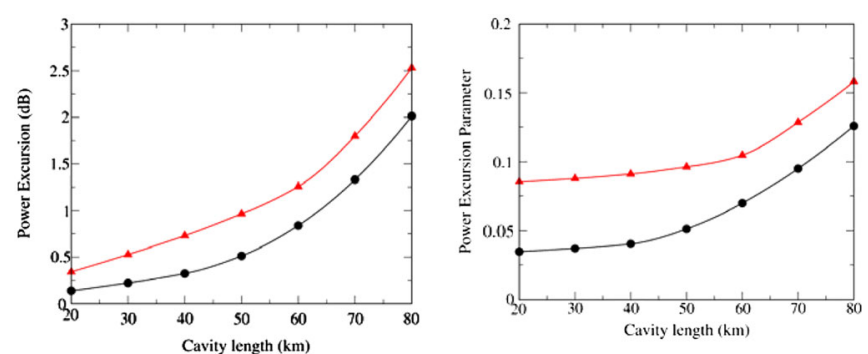

FIG. 4 (color online). Evolution of the power excursion (left panel) and $\delta P$ parameter (right panel) vs cavity length for $0 \mathrm{dBm}$ total signal power. Triangles, $20 \mathrm{~nm}$ bandwidth; circles, $10 \mathrm{~nm}$ bandwidth.

deleterious impact on power excursion. In any case, as illustrated by Fig. 4 (left), signal power excursion remains low over a broad bandwidth even for long cavities and experiences a similar growth both for 20 and $10 \mathrm{~nm}$ bandwidths. The evolution of $\delta P$ shows that the normalized reduction in power excursion remains nearly constant for cavity lengths of up to $40 \mathrm{~km}$, again showing a similar behavior over both 10 and $20 \mathrm{~nm}$ windows.

Conclusion.-We have demonstrated the possibility of achieving simultaneous cross-domain spatial-spectral transparency in ultralong fiber lasers. In our experiment, transparency was obtained at typical optical fiber communication wavelengths, using a $20 \mathrm{~km}$ cavity pumped with two single-wavelength sources at about $\sim 1365 \mathrm{~nm}$. With this setup, signal power excursions below $0.4 \mathrm{~dB}(\sim 7.8 \%$ intensity variation) over $20 \mathrm{~nm}$ and below $0.14 \mathrm{~dB}$ ( $\sim 3.1 \%$ intensity variation) over $10 \mathrm{~nm}$ were obtained. Our results show that such transparent links are resilient to high transmitted powers, which makes them attractive building blocks for multiwavelength nonlinear processing devices which could be described as nonlinear integrable systems without non-Hamiltonian dissipative terms. The achieved transparency could also allow for a substantial reduction of signal input power in transmission applications, suppressing nonlinear impairments. Our numerical predictions show that highly reduced power excursion over 10 and $20 \mathrm{~nm}$ bandwidths can also be achieved with links much longer than $20 \mathrm{~km}$, with potential applications to long-distance telecommunications.

[1] E. Snitzer, J. Appl. Phys. 32, 36 (1961).

[2] C. J. Koester and E. Snitzer, Appl. Opt. 3, 1182 (1964).

[3] R. H. Stolen, E. P. Ippen, and A. R. Tynes, Appl. Phys. Lett. 20, 62 (1972).

[4] K. O. Hill, Y. Fujii, D. C. Johnson, and B.S. Kawasaki, Appl. Phys. Lett. 32, 647 (1978).

[5] J. D. Ania-Castañón, Opt. Express 12, 4372 (2004).

[6] J. D. Ania-Castañón, T. J. Ellingham, R. Ibbotson, X. Chen, L. Zhang, and S. K. Turitsyn, Phys. Rev. Lett. 96, 023902 (2006).

[7] J. D. Ania-Castañón and S. K. Turitsyn, in Proceedings of the European Conference on Optical Communication (ECOC) (IEEE, New York, 2006), Vol. 7, p. 193.

[8] S. G. Grubb, T. Strasser, W. Y. Cheung, W. A. Reed, V. Mizrahi, T. Erdogan, P. J. Lemaire, A. M. Vengsarkar, D. J. Digiovanni, D. W. Peckham, and B.H. Rockney, in Optical Amplifiers and Their Applications, Technical Digest (Optical Society of America, Washington, DC, 1995), paper SaA4.

[9] T. J. Ellingham, J. D. Ania-Castañón, R. Ibbotson, X. Chen, L. Zhang, and Sergei K. Turitsyn, IEEE Photonics Technol. Lett. 18, 268 (2006).

[10] S. A. Babin, V. Karalekas, P. Harper, E. V. Podivilov, V. K. Mezentsev, J. D. Ania-Castañón, and S.K. Turitsyn, Opt. Lett. 32, 1135 (2007).

[11] V. Karalekas, J. D. Ania-Castañón, J. Pérez-González, X. Chen, L. Zhang, and P. Harper, Opt. Commun. 277, 214 (2007). 